\title{
La eficacia de los juegos de empresas en el ámbito de la Dirección de Empresas: Análisis de la experiencia con el ESIC Business Marketing Game
}

\author{
Miguel Blanco Callejo \\ Fernando García Muiña \\ Profesores de Organización de Empresas. Universidad Rey Juan Carlos.
}

\section{Resumen}

El objetivo del presente trabajo es analizar un fenómeno que está teniendo una importancia creciente en el ámbito del aprendizaje y la docencia universitaria dentro del área de Dirección Estratégica y Organización de Empresas en nuestro país: el empleo de juegos de simulación empresarial. En el marco de un creciente desarrollo y uso de las tecnologías de la información y las comunicaciones (TIC), el software de juegos de simulación empresarial constituyen una herramienta cuya explotación aún está pendiente de alcanzar todo su potencial.

Tomando como referencia una experiencia práctica de participación de los autores en un juego de empresas, el artículo pretende un acercamiento a la definición de los términos -a través de la delimitación de los conceptos de simulador empresarial y de juego de empresas- así como sus rasgos más característicos, su proceso de diseño y futuros desafíos.

\section{Palabras clave}

Juego de Empresas - Eficacia Docente - Organización de Empresas - Experiencia Empírica 


\section{Abstract}

The aim of this paper is to analyze the role of business simulation methodology in strategic management area. In an Information Technology growing development context, business gaming software constitutes an interesting tool, which potential is not completely exploited yet.

Based on our own experience as business players in ESIC’s Business Marketing Game, the paper tries two get two main objectives. Firstly, we seek to develop a complete theoretical framework; secondly, the paper assesses main applications these tools may have: 1) their use as learning tools for professors and 2) their role as a decision support system. After that, we present some key issues of these devices. Finally, we present conclusions and future research streams.

\section{Keywords}

Business Game - Teaching Efficacy - Management - Empirical Experience

\section{Delimitación del concepto de juego de empresas}

\subsection{El concepto de juego de empresas}

Diferenciación de los conceptos de simulador empresarial y juego de empresas

A modo de introducción, y antes de pasar a definir el concepto de juego de empresas, consideramos que resulta relevante la distinción entre dos conceptos -simuladores y juegos de empresa- que, a pesar de ser similares, han sido empleados indistintamente aunque de forma inapropiada, de acuerdo con Raia (1966) y Garras et al. (2002).

El primer matiz que distingue ambos conceptos es el grado de participación del usuario; de esta manera, los simuladores empresariales únicamente presentan al usuario un conjunto de información y relaciones dirigidas y controladas a través de un modelo algebraico basado en reglas lógicas. En el caso de los juegos de empresa, además del modelo, se requiere la participación del usuario mediante su interrelación con el sistema (Lane, 1995).

Otro matiz importante a la hora de diferenciar estos conceptos fue introducido por Keys y Wolfe (1990), y hace referencia al grado de verosimilitud con el que estos dispositivos 
representan la realidad. Una simulación es una secuencia especifica de actividades verosímiles, diseñada para la transmisión de conocimientos a los usuarios sobre el mundo real; por lo tanto, en este caso subyace que la verosimilitud del modelo es el aspecto clave determinante de su calidad; sin embargo, un juego se define como una secuencia específica diseñada con el objetivo fundamental de traspasar conocimientos a los jugadores para el desarrollo de la toma de decisiones, supeditando la verosimilitud del modelo a su eficacia en la transmisión de conocimientos (Lane, 1995).

Si se restringe la simulación al campo empresarial, ésta puede considerarse como un dispositivo que aporta información básica para la toma de decisiones en las organizaciones, que permite crear entornos en los que tienen lugar los procesos de aprendizaje, y en los que se pueden observar los comportamientos o decisiones directivas (Keys y Wolfe, 1990). La información básica que proporciona el modelo de simulación tratará de reflejar la realidad en la que las empresas desarrollan sus actividades. Las reglas que dirigen el modelo de simulación se formulan a partir de la observación y análisis del entorno empresarial real y su evolución (Schrieber, 1958).

\section{Concepto de juego de empresas}

Si bien tanto la estructura como el propio diseño de los juegos de empresa ha ido evolucionando a lo largo del tiempo, desde su aparición como herramienta soporte para la toma de decisiones ha existido un cierto consenso en cuanto a su conceptuación básica, y los autores han ido introduciendo diferentes matices al concepto.

De esta forma, una de las primeras aproximaciones al concepto de juego de empresas se realizó en el marco de una conferencia sobre el empleo de estos dispositivos en el ámbito universitario $^{1}$ : "Un juego de empresas es una situación artificialmente creada en la que varios jugadores han de tomar decisiones cada cierto tiempo en un entorno de negocio ficticio, que pueden afectar a las condiciones del entorno futuro. Además, las interacciones entre las decisiones y el entorno se determinan por un proceso de arbitraje que no está abierto a los jugadores”.

Otra propuesta de definición de juego de empresas es la de Greenlaw et al. (1962), quienes lo concibieron como un ejercicio de toma de decisiones estructurado en torno a un modelo de operaciones de negocio, en las cuales los participantes asumen el rol de gestores del negocio dentro del marco de la operación simulada.

\footnotetext{
${ }^{1}$ Actas de Conference on Business Games as Teaching Devices, Tulane University, 26-28 de abril, 1961, página 7.
} 
Por su parte, Babb et al. (1966: 466), lo definen como un "ejercicio de toma de decisiones en el que los equipos compiten entre sí, con la finalidad de alcanzar sus objetivos particulares”; es, por tanto, el aspecto competitivo entre los participantes del juego el matiz diferenciador que aparece en esta propuesta.

Para Shubik (1968) un juego de empresas es un ejercicio diseñado para resaltar las características más relevantes, tanto de las organizaciones (características internas de las empresas consideradas) como de su entorno (general y específico), que afectan a decisiones en todos los ámbitos de la empresa, y sobre las que los decisores ejercen un control directo, de forma que su eficacia queda medida en función de los resultados obtenidos.

En definitiva, como modelo de simulación, y tratando de ofrecer un concepto global que capte la totalidad de matices presentados en la literatura definimos un juego de empresas como un sistema de toma de decisiones en todos los ámbitos de la empresa -dentro de un contexto ficticio- que, en base a una serie de reglas de funcionamiento internas, permite a los participantes competir entre sí y evaluar la calidad de sus decisiones.

\subsection{Origen y evolución de los juegos de empresa}

El antecedente más remoto de los juegos de simulación empresarial se encuentra en juegos surgidos dentro del ámbito militar, los “juegos bélicos de estrategia” (war games), cuyas primeras manifestaciones aparecen en China e India aproximadamente hacia el año 3.000 a.c. Posteriormente, estos juegos fueron alcanzando un mayor grado de sofisticación, y fue a lo largo del siglo XIX cuando comienzan a utilizarse, de una forma más sistemática en el contexto de la formación militar, juegos/simuladores bélicos que pretendían ser simulaciones de la realidad. El empleo masivo de estos dispositivos como técnicas de aprendizaje tiene lugar durante el transcurso de la II Guerra Mundial (1939-1945); de este modo, un gran número de soldados americanos fueron entrenados y adiestrados mediante el empleo de este tipo de dispositivos. Tras la finalización del conflicto se comenzó a pensar en aplicar esta técnica para la formación dentro del ámbito empresarial.

A nivel académico, se acepta que el primer juego de gestión empresarial fue el Top Management Simulation creado por la AMA (American Management Association) en 1956 (Wolfe, 1976; Lane, 1995). Las universidades americanas de Carnegie Mellon y California Los Angeles (UCLA) fueron las pioneras y las que más potenciaron el empleo de estas herramientas (Bellman et al., 1957), a través del desarrollo de aplicaciones informáticas especializadas. 
A partir de los años 60 los juegos de empresas comenzaron a extender su aplicación masivamente en las escuelas de negocios y universidades de los Estados Unidos de América (Raia, 1966; Wolfe y Guth, 1975). A mediados de esta década los juegos de empresa se encontraban disponibles para prácticamente cualquier aspecto de la actividad corporativa y la estimación aproximada del mercado de estos dispositivos era de unos 100 millones de dólares al año (Neuhauser, 1976).

En 1975 se crea la Asociación para el Estudio de los Simuladores de Negocios y Aprendizaje Experimental (ABSEL) ${ }^{2}$, cuyo cometido es analizar el empleo de los juegos de simulación empresarial y su uso docente, y ha sido en el marco de los congresos organizados por esta asociación donde se puede hacer un seguimiento de la evolución de la investigación académica sobre la eficacia de estas técnicas docentes (Faria, 2000).

Durante la década de los años 80 y 90, la generalización del uso de los ordenadores personales, dentro de todos los ámbitos, especialmente a nivel educativo-formativo, fomentó el desarrollo y difusión de los juegos de empresa.

Los juegos de empresas constituyen una herramienta que consta de una doble dimensión docente e investigadora- y representan un instrumento con el que cuentan los profesores del área de Organización de Empresas. En el ámbito docente el debate sigue abierto en cuanto al nivel de eficacia de los juegos en comparación con otras alternativas docentes. Sin embargo, el empleo de estas herramientas como laboratorio de investigación para el proceso de toma de decisiones empresariales se está consolidando como un campo prometedor de análisis para la investigación científica futura (Toval y Flores, 1987; Mohiniyan, 1987).

\subsection{Características de los juegos de empresa}

La revisión de la literatura nos ha permitido identificar una serie de características básicas atribuibles a los buenos juegos de empresas (Bellman et al., 1957; Schrieber, 1958; Wolfe, 1997):

- Equilibrio entre estabilidad y aleatoriedad: los principios fundamentales sobre los que está construido el juego deben ser sólidos; no obstante, con la intención de incrementar el realismo del modelo, los juegos han ido introduciendo elementos estocásticos o aleatorios, de forma que las mismas decisiones pueden tener efectos diferentes, no solo como consecuencia

\footnotetext{
${ }^{2}$ Association for Business Simulation and Experiential Learning (ABSEL).
} 
del comportamiento de terceros agentes, sino también por efecto de los elevados niveles de dinamismo e incertidumbre del entorno empresarial (Cohen y Rhenman, 1961).

口 Sensibilidad: los resultados deben ser sensibles a los factores sobre los cuales está construido el modelo que sustenta el juego.

aquilibrio entre verosimilitud y facilidad de manejo: el grado de complejidad debe ser suficiente para reflejar la realidad de forma verosímil, pero sin poner en peligro su manejo por parte de los participantes. Wolfe (1997) demuestra que existe una relación positiva entre el grado de complejidad del juego y el nivel de aprendizaje, pero que dicha relación está limitada a cierto grado de complejidad.

- Adecuación a los objetivos perseguidos: el diseño del modelo debe contemplar ciertas restricciones (horarios de clase, disponibilidad de tiempo de directivos, tipos de decisiones objeto de simulación, evaluación de los resultados del juego, etc.)

\subsection{Tipología de los juegos de empresa}

El análisis crítico y comparado de distintos trabajos previos nos ha permitido identificar diversas tipologías de juegos de empresas, a partir de la consideración de diferentes criterios de clasificación. Así, uno de los más habituales es el que distingue entre juegos funcionales (Biggs, 1987; Cohen y Rhenman, 1961) y juegos integrales de dirección (Biggs, 1979; Horn y Cleaves, 1980; Keys, 1987; Keys y Biggs, 1990), según se concentren en una área funcional concreta de la organización o traten la empresa en su conjunto.

Otro criterio de clasificación de los juegos es el que atiende a su grado de realismo (Cohen y Rhenman, 1961). En este sentido, tal y como hemos apuntado anteriormente, con el paso del tiempo los juegos han ido introduciendo elementos estocásticos o aleatorios. De tal forma que se pueden distinguir entre juegos que se desarrollan en un ambiente de riesgo y certeza.

Junto a estos criterios referidos, Kriz (2003) presenta dos variantes de juegos de empresa: los juegos cerrados y los juegos abiertos. En los cerrados los jugadores reciben instrucciones claras basadas en reglas bien definidas. El problema se presenta a los participantes en un marco bien definido, y se espera que se resuelva de una manera orientada al objetivo previamente planificado, tan precisa como sea posible. En los juegos de forma libre, tanto el modelo de simulación como las reglas y los flujos del juego no son dados a priori. Sobre las bases del escenario inicial, los modelos del sistema se construyen por los propios jugadores. Los jugadores se convierten, por tanto, en agentes activos a la hora de diseñar las reglas de funcionamiento del modelo. 


\subsection{El proceso de construcción de los juegos: aspectos clave}

La construcción de un juego de empresas es un fenómeno complejo muy intensivo en información que pretende servir a la creación de conocimientos estratégicos por parte de sus usuarios.

Este proceso de creación del juego consta de varias fases (Figura 1)

- Fase 1: El juego trata de representar una parte de la realidad mediante su reducción a través de una serie de parámetros resumen.

- Fase 2: La aplicación de criterios docentes, didácticos y de carácter práctico aconsejan realizar una reducción de la realidad adicional.

- Fase 3: Esta fase concluye con el desarrollo de un prototipo del juego que permite que mediante el empleo de modelos de referencia de "participantes expertos” va a permitir realizar unas preliminares evaluaciones de su funcionamiento.

- Fases 4-5: Las dos últimas fases comparan la realidad con los escenarios representados en el juego y, a partir de la elaboración de informes comparativos entre ambos, lleva a cabo un proceso de continuo rediseño con el objetivo de optimizar la construcción y funcionamiento del juego de empresas.

Además la creación de un juego de empresas efectivo requiere fundamentalmente de tres habilidades por parte del equipo de diseño:

a Conocimiento de la materia $\rightarrow$ Dominio de los conocimientos que el juego pretende transmitir.

a Habilidad pedagógica $\rightarrow$ Comprensión de la naturaleza del proceso de aprendizaje que está siendo diseñado.

- Capacidad técnica $\rightarrow$ Capacidad para combinar los elementos físicos y el protocolo del proceso. 
Figura 1: Modelo para la creación de un juego de empresa en un entorno de aprendizaje efectivo.
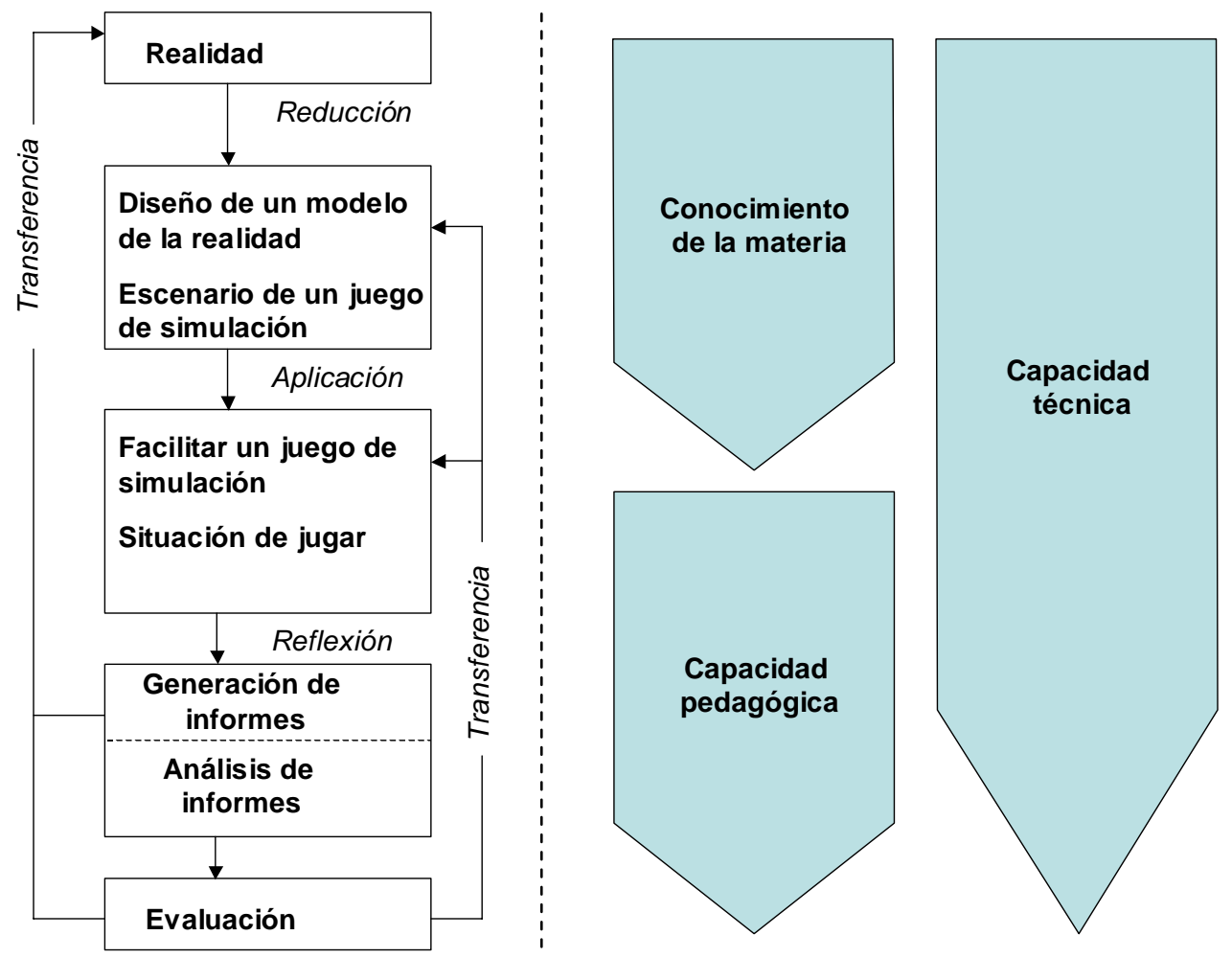

Adaptado de Kriz (2003)

Por último cabe destacar que la construcción de un juego de empresas eficaz exige a los diseñadores prestar atención sobre ciertos aspectos esenciales (Bellman et al., 1957):

口 Determinación del criterio básico sobre el que se toman las decisiones, esto es, base sobre la cual se determina lo que es una jugada óptima dentro de juego.

- La parametrización de los resultados de determinadas decisiones. Algunas relaciones son muy sencillas y el proceso causa-efecto puede apreciarse fácilmente; sin embargo, hay otras cuya relación causal resulta más complicada.

\subsection{Limitaciones de los juegos de empresas}

Antes de comenzar a profundizar en este aspecto cabe destacar, que en términos generales la literatura consultada presenta un cierto sesgo favorable al uso de esta herramienta, ya que la mayoría de autores resaltan sus ventajas, dejando en un segundo plano las limitaciones y problemas que presentan estos dispositivos. 
Merece la pena partir de la base de que algunas de las limitaciones que se perfilaban en los orígenes de los juegos de empresa (Bellman et al., 1957) han sido superadas gracias a desarrollos posteriores que han mejorado el rendimiento de estos dispositivos. Sin embargo, no debe olvidarse que los juegos constituyen siempre un intento de aproximación a la realidad y habrá algunas variables respecto de las cuales la alta dirección toma decisiones que quedan fuera del alcance de cualquier juego.

Uno de los aspectos que más se ha tenido en cuenta a la hora de analizar estos dispositivos desde el punto de vista de sus limitaciones ha sido, sin duda alguna, su coste. La literatura destaca el elevado coste que supone desarrollar un juego y advierte que es necesario conseguir la máxima explotación de los mismos para obtener rentabilidad de la inversión realizada (Bellman et al., 1957; Schrieber, 1958; Shubik, 1968).

Otro elemento a tener en cuenta es la dificultad de traducir a veces la información relativa a principios que son complejos de parametrizar dentro de un juego, como puede ser, por ejemplo, el efecto de la publicidad en la sensibilidad o la elasticidad de la demanda en el mercado. Shubik (1968) advierte del considerable riesgo de que a través de la mecanización que provoca el empleo de computadoras se convierta al juego en una mera normalización de conocimientos más o menos populares.

Uno de los aspectos que ha quedado recogido en la literatura es la aparición de una cierta desmotivación entre los usuarios de los juegos de empresa tras el entusiasmo inicial con el que normalmente se decide participar en estas actividades por parte de los jugadores. Esta situación se debe fundamentalmente a aspectos tales como lo tedioso y complejo de su manejo, la falta de adecuación del material de apoyo (Neuhauser, 1976) y el exceso de información que en ocasiones puede dificultar de forma innecesaria los procesos de definición de las estrategias y el análisis de los efectos de determinadas decisiones en los resultados (Greenlaw y Wyman, 1973). Esta situación suele provocar un desencanto progresivo de los jugadores, que suele tener efectos negativos sobre la eficacia del juego y provoca divergencias entre los objetivos teóricos de aprendizaje diseñados para él juego y el nivel de aprendizaje real de los usuarios (Fletcher, 1971; Neuhauser, 1976; Wolfe, 1997).

Además, la mayor capacidad para reflejar la realidad de los juegos de empresa frente al estudio de casos, que contrarrestaría el efecto de estos inconvenientes, no cuenta siempre con el respaldo empírico suficiente (McKenney, 1963; Boocock, 1966).

Para finalizar este apartado, Lane (1995) tratando de resumir las limitaciones de estos dispositivos sostiene que las principales críticas que se han realizado a los juegos de empresa se han 
centrado en aspectos tales como el grado de realismo de los simuladores, el excesivo énfasis dado al hecho de ganar el juego entre los participantes, la dificultad de planificación de tiempo, la falta de recompensas y castigos que motiven realmente a los participantes, la facilidad de empleo y, por último, la sobreexplotación que se ha hecho de la técnica.

\section{El empleo de los juegos de empresa}

Los juegos de empresa se han utilizado básicamente con dos grandes finalidades: 1) como laboratorios para el estudio del proceso de toma de decisiones empresariales (Bellman et al., 1957; Segev, 1987; Mohiniyan, 1987) y 2) como herramienta docente (Lane, 1995). Por ello, los usuarios de estas herramientas pueden ser desde alumnos universitarios o de escuelas de negocio (Schrieber, 1958) y directivos hasta investigadores del área de Organización de Empresas. Este epígrafe se dedica al análisis de los diferentes empleos que se ha dado a los juegos de empresa.

\subsection{Empleo de los juegos de empresa como un laboratorio para el análisis del proceso de toma de decisiones}

Uno de los aspectos que presenta mayor complejidad dentro del estudio de la Dirección Estratégica es el proceso mediante el cual se toman las decisiones por parte de la alta dirección de la empresa. Parte de la complejidad de estos procesos se debe a las importantes consecuencias que tienen las decisiones estratégicas dentro de las compañías.

Los juegos de empresa son herramientas que pueden proporcionar un marco adecuado a la hora de analizar la toma de decisiones como proceso, ya que dibujan una realidad simplificada, más sencilla, donde solamente se incluyen las variables relevantes y las consecuencias de las decisiones tomadas son ficticias. Desde un primer momento el diseño de los juegos de empresa ha estado orientado a la consecución de este objetivo (Bellman et al., 1957).

El modelo de Segev (1987) es un buen ejemplo de aplicación de los juegos al diseño y formulación de estrategias empresariales y su influencia en los resultados. Su principal ventaja es que permite controlar los factores externos, de forma que el contexto es homogéneo para todas las organizaciones. Así pues, los resultados empresariales dependen exclusivamente de la eficacia de las decisiones tomadas.

Mohiniyan (1987) defiende la utilización de los juegos, entendidos como colección sistemática de datos relativos a escenarios futuros, a los que los jugadores se han de enfrentar dando respuestas coherentes con sus conocimientos y con el escenario descrito. Esto permite analizar el proceso de toma de decisiones por parte de expertos en diversas materias, metodología que se 
conoce con el nombre de expert gaming. Centrándose en el caso de los juegos de empresa, analiza su empleo en el entorno empresarial de una industria; este escenario permite crear un laboratorio para el análisis del proceso de toma de decisiones por parte de los directivos. Sin embargo, entre sus conclusiones el autor destaca que el empleo de esta metodología requiere de un mayor volumen de investigación para la obtención de resultados más sólidos y generalizables.

\subsection{Empleo de los juegos de empresa como herramienta docente dentro del área de Dirección Estratégica}

Introducción: los juegos de empresa y el área de Dirección y Organización de Empresas

En la mayoría de los planes de estudio sobre Administración y Dirección de Empresas, el curso de Dirección Estratégica se encuentra al final de la licenciatura. La asignatura pretende ofrecer un conocimiento integral del funcionamiento de la empresa, centrándose principalmente en el nivel de las decisiones estratégicas. Para conseguir este propósito, el curso debe cubrir algunos aspectos que son fundamentales para el estratega (Wolfe y Guth, 1975):

a Familiarización con la naturaleza del entorno de negocio para la acción en la toma de decisiones.

• Oportunidad para la prueba y aplicación de conceptos y técnicas de Dirección Estratégica.

• Práctica en el análisis sistemático y la resolución de problemas complejos de negocio.

• El desarrollo de una visión de los negocios desde el punto de vista de la Alta Dirección.

Una de las grandes críticas que ha recibido la metodología docente relativa a esta área ha sido su excesivo enfoque teórico y la escasez de aplicaciones de carácter práctico. Es en este contexto en el que se justifica la difusión de los juegos de empresa, como una forma de aplicar en la práctica el gran número de conceptos aprendidos de forma teórica y abstracta.

De esta forma, el empleo de los juegos de empresa como una herramienta docente dentro del área de Dirección Estratégica ha estado presente desde su aparición, y actualmente se emplean en un gran número de instituciones educativas en todo el mundo (Lane, 1995). Sin embargo, a pesar de la visión optimista y entusiasta que presentan un buen número de trabajos que estudian el fenómeno (Schrieber, 1958; Cohen et al, 1960; Shubik, 1968; Wolfe y Guth, 1975; Wolfe, 1976; Lucht et al., 2003), no existe unanimidad en cuanto a su eficacia como herramienta docente. No obstante, en este sentido se considera ya que algunos estudios estaban sesgados debido a que parte de la investigación sobre juegos de empresa se basaba precisamente en opiniones de participantes y observadores directamente interesados (Raia, 1966); incluso, 
han aparecido ciertos trabajos que han llegado a poner en duda la efectividad de esta herramienta y su validez docente (Neuhauser, 1976).

\section{La efectividad docente de los Juegos de Empresa frente a otras técnicas}

Junto a la metodología habitual de la conferencia/lección magistral impartida por el profesor, se han empleado otras dos técnicas complementarias, especialmente indicadas para aproximar la práctica empresarial a la enseñanza: el estudio de casos y los juegos de empresa (Wolfe y Guth, 1975).

El uso del estudio de casos en el ámbito docente en Dirección Estratégica tiene su origen en la Escuela de Negocios de Harvard (Gordon y Howell, 1959), desde donde se extendió rápidamente al ámbito educativo de los Estados Unidos y posteriormente al resto del mundo.

La segunda gran herramienta son los juegos de empresa. Como ya se ha explicado anteriormente, estos dispositivos son una simulación de la realidad, diseñados para la consecución de una serie de propósitos docentes, como son: a) proporcionar experiencia en la toma de decisiones, b) conciliar diferentes puntos de vista entre los miembros de un mismo equipo y c) concebir el negocio como un todo incluyendo todas las áreas funcionales de la empresa (Schrieber, 1958).

Dado que la técnica de los juegos de empresa era más novedosa, uno de los esfuerzos fundamentales de la literatura ha consistido en demostrar que esta técnica era tan efectiva, sino más, que las anteriores (Wolfe, 1997; Faria, 2001). De hecho el trabajo de Faria y Nulsen concluye que los juegos de empresa representan la alternativa de aprendizaje que alcanza una mejor valoración frente al resto de métodos docentes (figura 2).

Figura 2: Valoración de la efectividad en el aprendizaje de métodos de enseñanza alternativos

\begin{tabular}{|c|c|}
\hline \multicolumn{1}{|c|}{ Método de enseñanza } & Valoración \\
\hline Juegos de Empresa & 7,9 \\
\hline Estudio de casos & 6,8 \\
\hline Conferencia/ Lección Magistral & 5,9 \\
\hline Libros de texto & 5,5 \\
\hline
\end{tabular}

Fuente: Adaptado de Faria y Nulsen (1996)

Sin embargo, parece existir un cierto acuerdo en la literatura acerca de que los juegos por sí mismos no son suficientes para el aprendizaje (Garris et al., 2002; Snow et al., 2002); siendo precisamente una de las principales fuentes de controversia el modo de medir el "éxito de la metodología”. Esta situación tiene como consecuencia la necesidad de emplear diferentes 
metodologías, que no tengan carácter excluyente sino complementario, y que permitan de forma conjunta conseguir todos los objetivos docentes. Wolfe y Rogé (1997:438) afirma que "las deficiencias en los juegos de empresa pueden ser compensadas mediante el empleo de otros métodos alternativos tales como casos específicos, conferencias de personas externas al curso, proyecciones o ejercicios experimentales” (Figura 3).

Figura 3: Eficacia docente: cuestión de ajuste

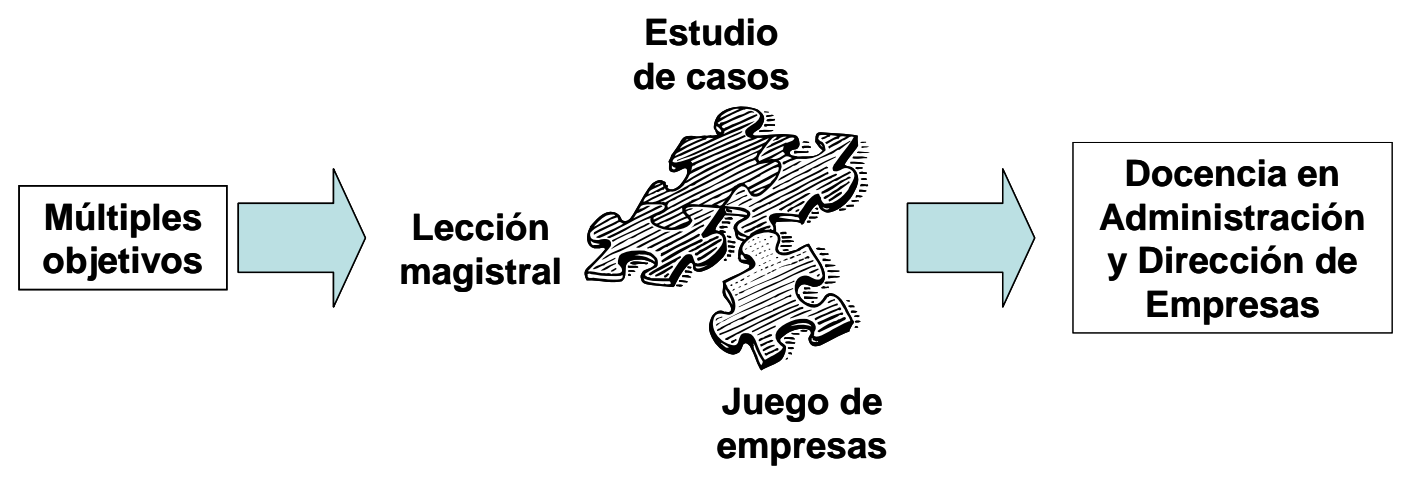

Fuente: Elaboración propia

Uno de los grandes problemas que presentan los métodos clásicos es que el conocimiento sobre las materias aprendidas permanece inactivo. La cultura predominante produce lo que se llama “conocimiento inerte”, que puede ser caracterizado como abstracto y artificialmente sistematizado y con escasa orientación a la aplicación práctica. Los juegos de empresa en este contexto podrían ser un medio de transformar el conocimiento en acción (Hsu, 1989), gracias a que los errores no tienen efecto en la realidad y pueden ser corregidos en la ficción del juego (Kriz, 2003).

¿Por qué han tenido éxito los juegos de empresa?

Los juegos de empresa basan teóricamente su éxito en dos aspectos fundamentales: a) su capacidad para despertar en los usuarios el interés y mejorar su satisfacción con el aprendizaje -a partir de la sensación de realismo y del espíritu de competitividad entre ellos- y b) su orientación hacia la práctica, es decir, 'hacer’ como mecanismo básico de aprendizaje según se actúa. Este segundo aspecto es de vital importancia, puesto que abre las posibilidades para aprender ciertos conocimientos tácitos de lenta y costosa acumulación que, a través de métodos clásicos de enseñanza, sería muy difícil (Burns y Gentry, 1992). El aprendizaje por la práctica promovido por estos sistemas de enseñanza se caracteriza por ser de aplicación directa al contenido, activo, interactivo, potenciar el contacto con el mundo real y autoevaluador de las decisiones tomadas. 
Fripr (1984) analiza la eficacia de los juegos de empresa como herramienta docente en función de los objetivos de aprendizaje perseguidos. Así, las fortalezas de esta metodología pueden ser resumidas en torno a dos planteamientos fundamentales. En primer lugar, la adecuación de este método como una forma de aprender por la práctica “learning by doing”³ (Certo, 1976; Fripr, 1984, Kolb y Fry, 1975). El aprendizaje por la práctica permite adquirir ciertos conocimientos tácitos de lenta y costosa acumulación que, a través de métodos clásicos de enseñanza, serían muy difíciles de conseguir (Burns et al., 1990). En segundo lugar, su capacidad para verificar las principales fases de los modelos de aprendizaje y adquisición de conocimientos más relevantes de la literatura como, por ejemplo, el proceso de aprendizaje experimental de Kolb y Fry (1975).

Figura 4: Fases del Modelo de aprendizaje experimental

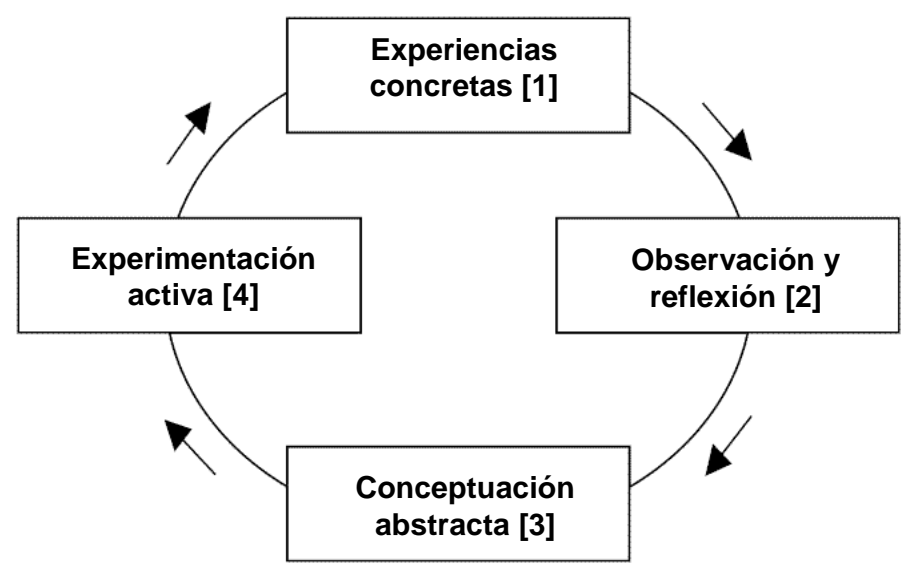

Fuente: Adaptado de Kolb y Fry (1975)

Kibbee, Craft y Nanus (1961) destacan que los juegos de empresas añaden dos elementos muy importantes a la aproximación a la Dirección Estratégica frente al estudio de casos: la objetividad del feed-back y el novedoso tratamiento de la dimensión tiempo.

La retroalimentación se convierte, por tanto, en uno de los pilares básicos sobre los que se apoya esta metodología de investigación y enseñanza. Los juegos de simulación proporcionan un rápido, concreto y consistente feed-back a las decisiones repetidas que se toman a lo largo del tiempo, y permiten la creación de laboratorios para contrastar modelos dinámicos de toma de decisiones empresariales.

\footnotetext{
${ }^{3}$ El acceso a experiencias prácticas resulta especialmente complicado en el caso de los directivos, en particular con carácter previo al desempeño de su actividad profesional
} 
En este sentido, diversos trabajos han estudiado la validez interna de la metodología de la simulación, en comparación con la eficacia de otros sistemas como el estudio de casos; sin obviar algunas limitaciones que se analizarán posteriormente, los resultados se suelen mostrar a favor del primero (Kaufman, 1976; McKenney, 1962, 1963; Raia, 1966 y Wolfe y Guth, 1975; Lucht et al., 2003). No obstante, las controversias acerca de la eficacia real de los sistemas de simulación aconsejan seguir profundizando en su estudio (Wolfe, 1976; Wolfe y Roberts, 1986; Norris y Snyder, 1982), en especial como consecuencia de las severas limitaciones metodológicas de la mayoría de trabajos empíricos que afrontan estas cuestiones (Mintu-Wimsatt y Meyers, 2002).

A diferencia del estudio de casos, los juegos de empresas plantean situaciones dinámicas a los participantes, cuya posición depende directamente de sus decisiones y de las de los demás -a parte de los efectos de la aleatoriedad que incluyen ahora ya la mayoría de juegos-. En el estudio de casos tradicional, se plantea una situación de una empresa ajena al jugador, quien no se siente responsable de haber llevado hasta ahí a la organización.

Pero la retroalimentación de los sistemas de simulación no es sino uno de sus diversos rasgos básicos. Junto a ella encontramos otros como son la caracterización del entorno a través de modelos lógicos o matemáticos (en algunos casos conocidos por los participantes y en otros casos no) o la interacción entre los jugadores y de éstos con el entorno (Cohen y Rhenman, 1961).

En definitiva, la literatura concluye que, a pesar de la discusión relativa a la forma de medir la variable dependiente éxito de la metodología, existen pruebas que avalan la efectividad de los juegos de empresa como vehículos útiles para la enseñanza en Dirección Estratégica, ya que producen incrementos significativos en el nivel de conocimiento de los participantes en comparación con otras técnicas docentes (Wolfe, 1997; Faria, 2001).

\section{El problema de la medida del éxito de los juegos de empresa}

En otras ocasiones el éxito se mide a través del nivel de satisfacción o actitudes de los alumnos tras su participación. No obstante, se considera que estos indicadores no están vinculados con el aprendizaje en sí mismo, sino con la experiencia de los alumnos con el juego (Remus, 1977; Estes, 1979; Remus y Jenner, 1981; Faria, 1986). Incluso, la evidencia empírica inicial -en el mismo sentido que otros estudios posteriores (Wolfe, 1997)- encuentra una falta de relación significativa entre el grado de satisfacción de los participantes y el nivel de aprendizaje (Catalanello y Brenenstuhl, 1977).

Finalmente, tampoco hay evidencia empírica que demuestre que aquellos grupos que han quedado mejor situados en el juego de empresas, hayan aprendido más, luego la posición con respecto a otros participantes debe quedar en un segundo plano a la hora de valorar el aprendizaje de los jugadores. Sin 
embargo, sí existen pruebas de que cuando se juega en grupo, frente a la participación individual, el aprendizaje es notablemente superior (Wolfe, 1997).

El perfil de usuarios de los juegos de simulación es relativamente amplio. En general podemos identificar dos perfiles básicos: el del directivo y el del alumno universitario que desea completar su formación a través de la simulación de situaciones más o menos parecidas a las que se tendrá que enfrentar a lo largo de su carrera profesional.

Aunque la importancia de esta herramienta es considerable para el desarrollo y formación de los directivos, existen muy pocos trabajos que adentren en estas cuestiones (Lucht et al., 2003). Quizá haya sido esta segunda aplicación la que ha recibido mayor atención en la literatura (Lucht et al., 2003; Faria, 1987; Wolfe, 1976). A pesar de la relevancia del estudio de los efectos de estas metodologías en la eficacia docente, el análisis de las ventajas e inconvenientes de los juegos de simulación necesita de más estudio para poder analizar de forma completa su idoneidad real.

Ventajas que presentan los juegos de empresa:

Una de las principales ventajas de los juegos de empresa es su capacidad para despertar en los participantes sus habilidades para la obtención y análisis de aquella información del entorno que resulte básica para sus decisiones. En la realidad empresarial ésta es la situación a la que se han de enfrentar los responsables de las organizaciones. Por tanto, a medida que un juego otorgue menos información de manera automática a los participantes, mayor será su complejidad y su capacidad para modelizar la realidad.

En este sentido, una de las mejores fuentes de información es la experiencia acumulada con el desarrollo del juego. Así, las decisiones normalmente no se realizan de forma aislada sino que existe una estrecha interacción entre las decisiones pasadas y futuras y entre los distintos agentes decisores (Babb et al., 1966).

Estas ventajas pueden explicar el empleo creciente de la tecnología de simulación en la docencia y formación en administración de empresas (Cohen y Lippert, 1999; Jonassen, 2000; Castleberry, 2001; Evans, 2001).

Como ventajas adicionales de los juegos de empresa se pueden citar las siguientes: (Lane, 1995)

• La posibilidad de aplicar los conocimientos teóricos adquiridos en la práctica, aunque sea virtual.

\ El contenido es descubierto por el propio usuario sin que haya ningún tipo de intermediario adicional.

๑ La experiencia es una de las fuentes de aprendizaje más potentes que existen. 
【 En ocasiones, el feed-back procedente de la simulación de la realidad puede ser más útil que el procedente de la realidad (por ejemplo, el parámetro tiempo, que lleva el saber las consecuencias de una decisión estratégica, etc).

Inconvenientes/limitaciones de adoptar estos dispositivos:

Entre los principales inconvenientes que se pueden presentar al emplear este tipo de herramientas: (Lane, 1995)

Falta de claridad en relación con los objetivos de aprendizaje.

ـ Dificultad para el diseño de materiales de apoyo eficacies para el aprendizaje.

• Exceso de atención en este método docente.

๑ Énfasis inapropiado sobre la tecnología.

\ Objetivos de aprendizaje demasiado ambiciosos (intentar que sea una herramienta de aprendizaje para demasiadas cosas).

\ Problemas en la presentación de los resultados de las decisiones anteriores.

a Falta de entrenamiento para los jugadores.

Además de todo esto, la implantación de estos dispositivos de simulación no está exenta de otras dificultades que desafían su éxito. Por un lado, el coste de las tecnologías puede frenar la adopción de estas tecnologías y, por otro lado, la complejidad para diseñar modelos reales puede conducir a la construcción de juegos de empresas poco eficaces alejados de la problemática empresarial (Lucht et al., 2003).

\section{Una experiencia empírica: EI ESIC Business Marketing Game}

\subsection{Descripción del juego}

El ESIC Business Marketing Game es un juego de empresas organizado por la Escuela Superior de Gestión Comercial y Marketing (ESIC), que consiste en la toma de una serie de decisiones en un mercado real simulado con productos reales que tienen consecuencias evaluadas por un software de simulación empresarial. Los participantes deben definir un Plan de Gestión y desarrollar el mismo en decisiones que abarcan todas las áreas básicas de la empresa. 
El simulador se enmarca en el seno de las actividades formativas que desarrolla el ESIC y el objetivo fundamental es conseguir acercar a los participantes a la toma de decisiones en el ámbito empresarial. La participación en el juego está abierta a estudiantes de postgrado y alumnos que se encuentren cursando los últimos cursos de la carrera universitaria y equipos senior formados por profesionales, trabajadores de empresas y organizaciones. La institución dispone de un amplio programa de becas que permiten, mediante el patrocinio de equipos por empresas, subvencionar a los equipos participantes ${ }^{4}$.

El juego presenta la simulación de un mercado que trata de reflejar una aproximación al negocio del patrocinador principal del juego. Durante la edición del 2005 el patrocinador principal fue Grupo Leche Pascual. El simulador por tanto se refería al mercado de productos lácteos (leche y yogurt) en tres mercados geográficos distintos y en un entorno competitivo donde podían encontrarse hasta cinco empresas competidoras.

El juego funciona a través de un proceso de eliminatorias que consta de cuatro fases y donde se van clasificando los equipos según el nivel de resultados financieros obtenidos en cada etapa. Los cinco primeros clasificados en el juego, reciben premios en metálico y la suscripción gratuita a cursos master y seminarios impartidos por el ESIC.

En el año 2005 se ha celebrado la décima edición de este juego de simulación empresarial. El juego presenta una participación creciente en número de participantes, así como en equipos inscritos en la competición (figura 5) ${ }^{5}$

Figura 5: Principales datos del ESIC Business Marketing Game

\begin{tabular}{|c|c|c|c|c|c|c|c|c|c|}
\hline \multicolumn{10}{|c|}{ EVOLUCIÓN DE MARKETING GAME } \\
\hline & 1996 & 1997 & 1998 & 1999 & 2000 & 2001 & 2002 & 2003 & 2004 \\
\hline & GONZALEZ & & & & GRUPO & DEUSTCHE & & & $\begin{array}{l}\text { GRUPO } \\
\text { LECHE }\end{array}$ \\
\hline Patrocinador Principal & BYASS & ESIC & VIA DIGITAL & EURODOMO & PRO\&SALES & BANK & VODAFONE & FESTINA & PASCUAL \\
\hline Empresas colaboradoras & 164 & 126 & 131 & 172 & 196 & 163 & 185 & 145 & 133 \\
\hline Equipos inscritos & 230 & 210 & 235 & 230 & 248 & 285 & 305 & 315 & 320 \\
\hline Participantes & 920 & 850 & 910 & 1020 & 1110 & 1147 & 1258 & 1270 & 1345 \\
\hline
\end{tabular}

\subsection{Valoración global del juego}

\footnotetext{
${ }^{4}$ Fuente: “Vuelve Business Marketing Game”, ABC Nuevo Trabajo Economía y Empresas, 7 de diciembre de 2003.

${ }^{5}$ Fuente: “Mas de trescientos equipos se han apuntado a Marketing Game 2004”, ABC Nuevo Trabajo Economía y Empresas, 1 de febrero de 2004.
} 
Siguiendo el esquema básico de las propiedades que debe cumplir un juego de empresas eficaz, en el caso de ESIC Business Marketing Game podemos llegar a las siguientes conclusiones:

口 $\quad$ Equilibrio entre estabilidad y aleatoriedad $\rightarrow$ Entendemos que no cumple con este requerimiento ya que el desarrollo del juego en muchas ocasiones distaba de reflejar lo que podría considerarse una evolución normal del mercado. Parece que el criterio que rige en el funcionamiento del juego es la aleatoriedad, que en ocasiones parece tener un peso excesivo en el desarrollo de las decisiones del mismo. Cabe destacar que en ciertas condiciones se produce un cambio bastante importante en el mercado sin una explicación clara de los motivos, lo que lleva a pensar en la primacía de la aleatoriedad sobre la estabilidad en el juego.

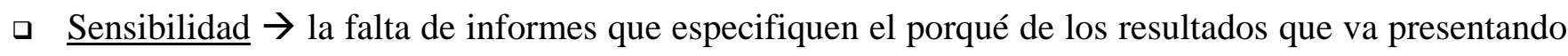
el juego en sus diversas fases hace que sea difícil de valorar la sensibilidad de los resultados ante diversos factores. En ocasiones los informes que facilita el programa no dejan clara la influencia de variables tales como la inversión en publicidad, o el grado de exposición del cliente al producto, o la sensibilidad de la demanda ante modificaciones de precios en los resultados conseguidos por la compañía.

a Equilibrio entre verosimilitud y facilidad de manejo $\rightarrow$ las instrucciones del juego son bastante completas y detalladas permitiendo afrontar el juego con el conocimiento suficiente para desarrollar la estrategia concebida por los participantes. Sin embargo cabe destacar la necesidad de contar con herramientas informáticas de apoyo tales como ficheros en formato de hoja de cálculo que permitan agilizar el proceso de toma de decisiones por parte de los participantes que, en ocasiones, requieren de un notable esfuerzo para su construcción y mantenimiento. No obstante es algo que los jugadores deben afrontar como algo lógico para el buen desarrollo del juego.

Otro aspecto que merece la pena resaltar, en relación con este aspecto, es que el diseño del programa hace que en ocasiones se comentan errores que tienen como consecuencia graves pérdidas para los participantes que carecen de ninguna correspondencia con situaciones que podrían darse en la realidad y que podrían ser fácilmente subsanables a través de un sistema de bloqueos dentro del programa (por ejemplo, el juego es capaz de aceptar una decisión en la cual no se decide fabricar cuando en la realidad esta decisión sería imposible).

- Adecuación a los objetivos finales del juego: tras el profundo análisis que debe realizarse de las instrucciones al empezar el juego, la toma de una decisión a la semana hace muy asequible su seguimiento.

\section{3.- Valoración global de los juegos de empresa: algunas reflexiones finales}


En el marco de la enseñanza en el campo de la administración de empresas resulta particularmente complicada la aplicación práctica de los conocimientos a la toma de decisiones. Es quizás en este aspecto en el que los juegos de empresa presentan una aportación de mayor interés, ya que permiten al alumno entender algunos de los conceptos que resultan abstractos a través de las explicaciones teóricas, como puede ser el caso del funcionamiento de una empresa como sistema o la interrelación entre las diferentes partes de la empresa y el entorno que la rodea. Por lo tanto, estos dispositivos permiten al alumno/usuario comprender a grandes rasgos el funcionamiento real de una compañía que se encuentra compitiendo en un mercado y las diferentes decisiones que deben ser tomadas por los directivos para conseguir que la empresa tenga éxito.

El juego genera grandes expectativas en los participantes pero dichas expectativas se ven defraudadas, en parte, cuando comienza a apreciarse que los resultados del juego no siempre responden a lo que debería ser un comportamiento racional, académico y teórico, aprendido en el marco de la enseñanza en cursos de grado superior o escuelas de negocio, y es que la aleatoriedad del entorno puede que no se haya trasladado bien a los programas informáticos. Esto tiene como consecuencia, en primer lugar, que se limita la capacidad docente de la herramienta para transmitir conocimientos a los participantes. En segundo lugar se desmotiva de forma importante a los usuarios, al no ver reflejadas en el juego las consecuencias esperadas de decisiones racionales y la falta de explicaciones convincentes de los motivos que conducen a la obtención de determinados resultados.

Por tanto todo juego de empresas debería contar con un servicio de asistencia y seguimiento y generación automática de informes, que además de ayudar a resolver los problemas técnicos que pudieran surgir durante el desarrollo del juego, fuera capaz de justificar las respuestas de la computadora ante las decisiones “racionales” de los participantes. De lo contrario, el juego pierde toda la perspectiva docente para el usuario y además se corre el riesgo de convertir la participación en un simple juego de azar en el que el usuario toma decisiones de forma automática a la espera de la respuesta mecánica en función de un algoritmo matemático.

Quizás este problema podría solucionarse introduciendo dentro del programa el concepto de adquisición de información más completa que los informes habitualmente ofrecidos en el marco de los juegos, que se limitan a ser meras descripciones de la situación sin aportar ninguna clase de elementos justificativos del porqué de las consecuencias de determinadas decisiones. Si no se incluye el traspaso de información como medio básico de aprendizaje, el objetivo fundamental de difundir conocimientos a los jugadores, para el desarrollo y toma de decisiones, no se verá cumplido, y el juego se limitará a ser un simple modelo de simulación. 


\section{Conclusiones y líneas futuras de investigación}

A modo de conclusión, a partir de una experiencia práctica de participación de los autores en un juego de empresas, en este trabajo hemos enfrentado, en primer lugar, los conceptos de simulador empresarial y de juego de empresas, En segundo lugar, nos hemos adentrado en la eficacia docente de los juegos de empresas y su utilidad como instrumento para mejorar los procesos de toma de decisiones directivas y, finalmente, hemos presentado una valoración global sobre los aspectos más destacados tras la experiencia de haber participado en el Juego de Simulación empresarial ESIC Business Marketing Game.

A pesar de ello, siguen quedado muchos aspectos por analizar siendo, desde nuestro punto de vista, la medición de la variable representativa del éxito de las metodologías docentes el principales interés de la investigación futura, si se desea llegar a concretar la mayor o menor eficacia de cada una de las alternativas, no tanto como medidas excluyentes, cuanto como metodologías complementarias, que permitan mejorar la docencia y los sistemas de aprendizaje en nuestras aulas.

\section{Bibliografía}

- $\quad$ BELLMAN R, CLARK C.E.; MALCOM D.G; CRAFT C.J; RICCIARDI. F.M (1957): “On the Construction of a Multi-Stage, Multi-Person Business Game”, Operations Research, Vol. 5, nº, Aug 1957, pp 469-503

- BOOCOCK, S.S. (1966): “An Experimental Study of the Learning Effects of Two Games with Simulated Environments”, American Behavioral Scientist, Vol. 10 (2), pp. 8-18.

- COHEN K.J (1960): “The Carnegie Tech Management Game”, Journal of Business, 33, October 1960, pp 304

- FARIA A.J. (1987): “A survey of the use of business games in academia and Business”. Simulation and Games, Vol. 18 (2), pp 207-224.

- FARIA A.J. (2001):“The changing nature of business simulation/ gaming research: A brief story”, Simulation \& Gaming, March 2001, pp 97-110.

- $\quad$ FARIA, A.J.; NULSEN, R. (1996): "Business Simulation Games: Current Usage Levels: A Ten Years Update”, Developments in Business Simulation \& Experiential Exercises, Vol. 23, pp. 23-28.

- $\quad$ FLETCHER, J.L. (1971):“The Effectiveness of Simulation Games as Learning Environments: A Proposed Program of Research”, Simulation and Games, Vol. 5 (4), pp. 425-454.

- GARRIS R; AHLERS R.; DRISKELL J.E. (2002):“Games, Motivation and learning: A research and practice model”, Simulation \& Gaming, Vol. 33, No 4, pp 441-467.

- GREENLAW P.S; HERRON L.W. RAWDON R. (1962): "Business Simulation”, Englewood Cliffs: Prentice Hall.

- GREENLAW, P.S.; WYMAN, F.P. (1973): “The Teaching Effectiveness of Games in Collegiate Business Course”, Simulation and Games, Vol. 4 (3), pp. 259-294.

- GORDON R.A; HOWELL J.E (1959): “Higher Education for Business”, New York, Columbia University Press, pp 207. 
- HSU E. (1989): "Role-event gaming simulation in management education: A conceptual framework and review”. Simulation and Games: An international journal, Vol. 4(1), pp 409-438.

- $\quad$ KIBBEE J.M.; CRAFT C.J.; NANUS B. (1961): “Management Games: A new Technique for Executive Development” (New York; Reinhold Publishing Corp, pp 41.

- KEYS B.; WOLFE J (1990):“The Role of Management Games and Simulations in Education and Research”, Journal of Management, Vol. 16, No 2, pp 307-336.

- $\quad$ KRIZ W.C. (2003):“Creating effective learning environments and learning organizations through gaming simulation design”, Simulation and Gaming, Vol. 34, No 4, pp 495-511.

- LANE D.C. (1995):“On a Resurgence of Management Simulations and Games”, The Journal of the Operational Research Society, Vol. 46, No 5, pp 604-625.

- McKENNEY, J.L. (1963):“Evaluation of Decision Simulation as a Learning Environment”, Management Technology, Vol. 3 (1), pp. 56-57.

- MOYNIHAN P. (1987):“Expert Gaming: A means to Investigate the Executive Decision Process”, The Journal of the Operational Research Society, Vol. 38,No 3, pp 215-231.

- $\quad$ NEUHAUSER J.J. (1976):“Business Games Have Failed”, The Academy of Management Review, Vol. 1, No 4, pp 124-129.

- $\quad$ RAIA A.P. (1966): “ A study of the educational value of games”, Journal of Business, Vol. 39, Issue 3, pp 339-352

- $\quad$ SCHRIEBER A.N. (1958):“The Theory and Application of the Management Game Approach to Teaching Business Policy”, The Journal of the Academy of Management, Vol. 1,No 2, pp 51-57.

- $\quad$ SEGEV E. (1987):“Strategy, Strategy-Making, and Performance in a Business Game”, Strategic Management Journal, Vol. 8, No 6, pp 565-577.

- $\quad$ SHUBIK M. (1968):“Gaming: Costs and Facilities”, Management Science, Vol. 14, No 11, Theory Series pp 629660.

- $\quad$ SNOW S; GEHLEN F.; GREEN J.C (2002):“Different ways to introduce a business simulation: The effect on student performance”, Simulation \& Gaming, Vol. 33, No 4, pp 526-532.

- $\quad$ TOVAL A; FLORES M. (1987): “Computer systems simulation and education: description of an experience”, Computers and Education, Vol. 11, 293-303.

- $\quad$ WOLFE J; GUTH G.R. (1975):“The Case Approach vs. Gaming in the Teaching of Business Policy: An experimental Evaluation”, The Journal of Business, Vol. 48, No 3, pp349-364.

- WOLFE J. (1976):“The effects and effectiveness of simulations in business policy teaching applications”, Academy of Management Review, April 1976, pp 47-56.24

- WOLFE J. (1997):“The effectiveness of business games in strategic management course work”, Simulation and Gaming: An interdisciplinary Journal, Vol. 16(2), pp 251-288.

- $\quad$ WOLFE J; FRITZSCHE D.J. (1998):“Teaching Business Ethics with Management and Marketing Games”, Simulation and Gaming, Vol. 29, No 1, pp 44-59. 